Appeared in Chaos, Solitons \& Fractals, 6, 399-410 (1995). Special volume: Complex Systems in Computational Physics. Preprint LN-2 (10-10-93). Final version: 20-03-95.

\title{
Scale Relativity: From Quantum Mechanics to Chaotic Dynamics
}

\author{
LAURENT NOTTALE
}

CNRS, DAEC, Observatoire de Paris-Meudon, F-92195, Meudon Cedex, France

\begin{abstract}
Scale relativity is a new approach to the problem of the origin of fundamental scales and of scaling laws in physics, which consists in generalizing Einstein's principle of relativity to the case of scale transformations of resolutions. We recall here how it leads one to the concept of fractal space-time, and to introduce a new complex time derivative operator which allows to recover the Schrödinger equation, then to generalize it. In high energy quantum physics, it leads to the introduction of a Lorentzian renormalization group, in which the Planck length is reinterpreted as a lowest, unpassable scale, invariant under dilatations. These methods are successively applied to two problems: in quantum mechanics, that of the mass spectrum of elementary particles; in chaotic dynamics, that of the distribution of planets in the Solar System.
\end{abstract}

\section{INTRODUCTION}

This contribution is a good opportunity to provide the reader with a summary of the present status of the theory of scale relativity, and to develop in more detail some of its consequences. A full description of the motivations and the premises to this new approach can be found in Ref [1].

One of the main open problems of today's physics is that of the nature of fundamental scales and of understanding scale-dependent phenomena. We have proposed to construct a theory which could deal with such questions by generalizing Einstein's principle of relativity to the case of scale transformations. Namely, we introduce resolution as an essential variable that characterizes the state of scale of reference systems, and we require that the laws of physics apply to all reference systems whatever their state (of motion, which yields standard relativity, but also of scale). This principle is mathematically translated into the requirement of scale covariance of the equations of physics under resolution transformations (contractions and dilations).

This approach leads to the suggestion that the quantum space-time should be described as a non-differentiable fractal continuum [2-4]. Such a concept of quantum fractal space-time finds its origin in early attempts to describe in a geometric way the typical paths of a quantum particles [5-7], in particular using the concept of fractals as introduced by Mandelbrot [8], and has known a recent burst of interest [1,9-11]. One of the straighforward consequence of the fractal nature of space-time would be that, since there are an infinity of geodesical lines between any couple of points in such a space-time, the description of the trajectories of particles becomes 
necessarily probabilistic. One can demonstrate that the fractal hypothesis leads one to introduce a double Wiener process à la Nelson $[12,13]$, whose effect can be reduced to the replacement, in the equations of mechanics, of the classical time-derivative by a new complex covariant derivative [1,14]. But the most profound consequences of scale relativity concern high energy particle physics: indeed we have suggested to replace the present renormalization group, whose mathematical structure is that of the Galileo group, by a Lorentzian renormalization group $[1,14,15]$. Such a theory leads to a reinterpretation of the Planck length-scale, which would now play for scale laws a role similar to that of the velocity of light for motion laws (limiting, unpassable scale, invariant under dilatation) and solves the problem of divergence of masses and charges in the quantum theory [14].

In the present contribution, after having specified the relation between nondifferentiable continuity and fractality (in the general meaning of scale-divergence), we generalize this method and apply it to two apparently disconnected domains: quantum mechanics and chaotic dynamics.

\section{RELATION BETWEEN NONDIFFERENTIABILITY AND SCALE DIVERGENCE}

One of the main argument in favor of the idea that scale relativity might be a fundamental principle of physics is the profound link that exists between nondifferentiability and scale-divergence. This relation has been demonstrated in Ref [1], but it needs further analysis. One can actually establish the following theorem:

A continuous and nowhere differentiable function drawn between two points separated by a finite distance in the Euclidean plane has an infinite intrinsic length; such a function depends explicitly on the resolution $r$, and its length is such that $\mathcal{L}(r) \rightarrow \infty$ when $r \rightarrow 0$. In other words, it is fractal (in a general meaning [8]).

What about the reverse proposition: Is a continuous function whose length is scale-divergent between any couple of points (such that $x_{\mathrm{A}}-x_{\mathrm{B}}$ finite), i.e. $\mathcal{L}(r) \rightarrow \infty$ when $r \rightarrow 0$, nondifferentiable ? The answer is as follows:

If the length diverges as fast as, or faster than a power law, i.e. $\mathcal{L}(r) \gtrsim(\lambda / r){ }^{\delta}$, (i.e., standard fractal behavior) then the function is certainly nondifferentiable; in the intermediate domain of slower divergences (for example, logarithmic divergence, $\mathcal{L}(r) \propto \ln (\lambda r)$, etc...), the function may be either differentiable or nondifferentiable.

A complete demonstration of this theorem and its generalization to fractal space is too long to be given in this short paper and will be presented elsewhere.

\section{GENERALIZED SCHRÖDINGER'S EQUATION}

We have indicated in Refs [1,14] how giving up the hypothesis of differentiability of space (then of particle trajectories) naturally leads to quantum mechanics (i.e., to defining a complex probability amplitude that satisfies the Schrödinger equation). Here we shall first recall the main steps of this demonstration, then also relax some of the assumptions.

We assume that space is continuous and nondifferentiable, so that the geodesics of such a space are also expected to be continuous, nondifferentiable and in infinite number between any couple of points. Note that we consider here only the one-particle, nonrelativistic case, but our results may be generalized to the $n$-particles and 
relativistic case (Klein-Gordon and Dirac equations) [16]. This can be expressed by describing the position vector of a particle by a finite, continuous fractal 3-function $\boldsymbol{x}(t, \delta t)$, explicitly dependent on the time resolution $\delta t$. Nondifferentiability also implies that the variation of the position vector between $t-d t$ and $t$ and between $t$ and $t+d t$ is described by two a priori different processes:

$$
\begin{aligned}
& x(t+d t, d t)-\boldsymbol{x}(t, d t)=\boldsymbol{b}_{+}(\boldsymbol{x}, t) d t+\boldsymbol{\zeta}_{+}(t, d t)\left(d t / \tau_{\mathrm{o}}\right)^{1 / D} \\
& \boldsymbol{x}(t, d t)-\boldsymbol{x}(t-d t, d t)=\boldsymbol{b}_{-}(\boldsymbol{x}, t) d t+\boldsymbol{\zeta}_{-}(t, d t)\left(d t / \tau_{\mathrm{o}}\right)^{1 / D}
\end{aligned}
$$

where $\boldsymbol{b}_{+}$is an average forward velocity and $\boldsymbol{b}_{-}$an average backward velocity, and where $D$ is the fractal dimension of the trajectory.

Let us define, following Nelson $[12,13]$, mean forward and backward derivatives, $d_{+} / d t$ and $d_{-} / d t$ :

$$
\frac{d_{ \pm}}{d t} \boldsymbol{y}(t)=\lim _{\Delta t \rightarrow 0 \pm}<\frac{y(t+\Delta t)-y(t)}{\Delta t}>\text {, such that } \frac{d_{+}}{d t} \boldsymbol{x}(t)=\boldsymbol{b}_{+} ; \frac{d_{-}}{d t} \boldsymbol{x}(t)=\boldsymbol{b}_{-} .
$$

We have suggested [1] to combine the forward and backward derivatives of (3.2) in terms of a complex derivative operator

$$
\frac{d}{d t}=\frac{\left(d_{+}+d_{-}\right)-i\left(d_{+}-d_{-}\right)}{2 d t}
$$

which, when applied to the position vector, yields a complex velocity [1]

$$
v=\frac{d}{d t} \boldsymbol{x}(t)=\boldsymbol{V}-i \boldsymbol{U}=\frac{\boldsymbol{b}_{+}+\boldsymbol{b}_{-}}{2}-i \frac{\boldsymbol{b}_{+}-\boldsymbol{b}_{-}}{2} .
$$

The next step consists in jumping to a statistical representation. It is easy to show that the position vector $\boldsymbol{x}(t)$ can be now assimilated to a stochastic process which satisfies the following relations (respectively for the forward $(d t>0)$ and backward $(d t<0)$ process):

$$
d \boldsymbol{x}(t)=\boldsymbol{b}_{+}[\boldsymbol{x}(t)] d t+d \boldsymbol{\xi}_{+}(t)=\boldsymbol{b}_{-}[\boldsymbol{x}(t)] d t+d \boldsymbol{\xi}_{-}(t)
$$

where the $d \xi(t)$ 's have mean zero, $\left\langle d \xi_{ \pm} i>=0\right.$, and satisfy

$$
<d \xi_{ \pm} i d \xi_{ \pm}>= \pm 2 \mathscr{D} \delta_{i j} d t^{2 / D}
$$

In previous papers, only the case of fractal dimension $D=2$ and of a constant "diffusion coefficient" $\mathscr{D}$ was considered in detail. This case corresponds to a twin Markov-Wiener process in which the $d \xi(t)$ 's are mutually independent, and yields the Schrödinger equation and quantum mechanics $[1,14]$. However, two generalizations are particularly relevant: the case of a fractal dimension becoming different from 2 (as suggested by the development of scale relativity, see Refs $[1,14,15]$ and below), and the case of a diffusion coefficient varying with position and time, $\mathcal{D}=\mathscr{D}(x, t)$, that must be considered in applications of this approach to chaotic dynamics. The general problem of tackling properly the case $D \neq 2$ lies outside the scope of the present contribution: such processes correspond to fractional Brownian motions, which are known to be non-Markovian, and persistent $(D<2)$ or antipersistent 
$(D>2)$. We shall consider only the case when the fractal dimension $D$ is close to 2 . Indeed, in this case its deviation from 2 can be approximated in terms of an explicit scale dependence on the time resolution, as first noticed by Mandelbrot and Van Ness [17]. Namely we write:

$$
<d \xi_{ \pm} i d \xi_{ \pm j}>= \pm 2 \mathcal{D}^{\prime}(x, t) \delta_{i j} d t \quad(\delta t / \tau)^{(2 / D)-1}
$$

where $\tau$ is some characteristic time scale. Hence the effect of $D \neq 2$ can finally be dealt with in terms of a generalized, scale-dependent, "diffusion" coefficient:

$$
\mathcal{D}=\mathcal{D}(x, t, \delta t)=\mathscr{D}^{\prime}(x, t)(\delta t / \tau)^{(2 / D)-1} .
$$

Let us now proceed further, following the lines of Ref [14]. The complex time derivative operator (3.4) is found to be given by the following expression [1]:

$$
\frac{d}{d t}=\frac{\partial}{\partial t}+\mathcal{V} \cdot \nabla-i \mathscr{D} \Delta
$$

We shall now use our postulate that the passage from classical (differentiable) mechanics to the new nondifferentiable mechanics that is considered here can be implemented by a unique prescription: Replace the standard time derivative $d / d t$ by the new complex operator d/dt. In other words, d/dt plays the role of "scalecovariant derivative". Let us indicate once again the main steps by which one may generalize classical mechanics using this new covariance.

We assume that any mechanical system can be characterized by a Lagrange function $\mathcal{L}(\boldsymbol{x}, \mathcal{V}, t)$, from which a complex action $S$ is defined, $S=\int \mathcal{L}(x, \mathcal{V}, t) d t$. The stationary-action principle, applied on this new action with both ends of the above integral fixed, leads to generalized Euler-Lagrange equations [1]

$$
\frac{d}{d t} \frac{\partial \mathcal{L}}{\partial \mathcal{V}}=\frac{\partial \mathcal{L}}{\partial \boldsymbol{x}}
$$

Assuming homogeneity of space in the mean leads to defining a complex momentum $P=\frac{\partial \mathcal{L}}{\partial \mathcal{V}}=\nabla S$.

We now specialize and consider Newtonian mechanics. The Lagrange function of a closed system, $L=\frac{1}{2}$ $m v^{2}-v$, is generalized as $\mathcal{L}(\boldsymbol{x}, \boldsymbol{v}, t)=\frac{1}{2} m \boldsymbol{v}^{2}-\mathcal{v}$. The Euler-Lagrange equations keep the form of Newton's fundamental equation of dynamics

$$
-\nabla v=m \frac{d}{d t} v
$$

which is now written in terms of complex variables and time derivative operator. This is a complex generalisation of the Nelson-Newton equation [12]. The complex momentum $\mathscr{P}$ now reads $\mathscr{P}=m \mathcal{V}$, so that we arrive at the conclusion that, in this Newtonian case, the complex velocity $v$ is a gradient, namely the gradient of the complex action, $v=\nabla S / m$.

Up to now, the various above steps do not differ from that of the standard Wiener-Markov situation. This is no longer the case in the following steps, since we must account for the diffusion coefficient being now a function of 
position.

Rather than defining, as in [1,14], the probability amplitude by the relation $\psi=e^{i S / 2 m \mathcal{D}}$, we define an average value of the diffusion coefficient, $\langle\mathscr{D}\rangle$, and we introduce the complex function $\psi$ from the relation,

$$
\psi=e^{i S / 2 m<\mathscr{D}>}
$$

Then $\psi$ is related to the complex velocity :

$$
\mathcal{V}=-2 i<\mathscr{D}>\nabla(\ln \psi)
$$

Our generalized Newton equation (3.11) now takes the form

$$
\nabla \mathbb{U}=2 i m<\mathcal{D}>\frac{d}{d t}(\nabla \ln \psi)
$$

We must remain aware that $d$ and $\nabla$ do not commute, and that $\mathcal{D}$, which appears in the expression for $d / d t$, is now a function of $x$. The presence in the calculation of both $\mathscr{D}$ and its mean $<\mathscr{D}>$ leads us to introduce the notation:

$$
\mathcal{D}(x, t)=\langle\mathcal{D}>+\delta \mathcal{D}(x, t),
$$

where $\langle\mathscr{D}>$ is a constant respectively to variables $x$ and $t$, but may include an explicit scale-dependence in terms of time resolution $\delta t$. Equation (3.14) may be finally given the form of a generalized Schrödinger equation:

$$
\nabla\left\{\frac{\mathscr{V}}{2 m<\mathscr{D}>}-\frac{1}{\psi}\left[\mathcal{D} \Delta \psi+i \frac{\partial \psi}{\partial t}\right]+\delta \mathscr{D}(\nabla \ln \psi)^{2}\right\}=-\nabla(\delta \mathscr{D}) \Delta \ln \psi
$$

We let open for future works the study of the general form of this equation. We shall only consider here some special, simplified cases:

(i) $\mathscr{D}=$ cst $=\hbar / 2 m$ : in this case $\delta \mathscr{D}=0$, and the last two terms disappear. The equation can be integrated and yields the Schrödinger equation $[1,14]$.

(ii) $\mathscr{D}=\mathscr{D}(\delta t)=\mathscr{D}^{\prime}(\delta t / \tau)^{(2 / D)-1}$ : this case corresponds to a diffusion coefficient which remains a constant in terms of positions and time, but which includes the effect of a fractal dimension different from 2 . The last two terms of (3.16) also disappear in this case, so that we still obtain a Schrödinger-like equation:

$$
\mathscr{D}^{2}(\delta t) \Delta \psi+i \mathcal{D}(\delta t) \frac{\partial \psi}{\partial t}=\frac{\mathcal{\psi} \psi}{2 m}
$$

The behavior of this equation is in agreement with the underlying stochastic process being no longer Markovian for $D \neq 2$. The "ultraviolet" $(\delta t<<\tau)$ and "infrared" $(\delta t>\tau)$ behaviors are reversed between the cases $(D<2)$ and $(D>2)$, but anyway they correspond to only two possible asymptotic behaviors, $\mathscr{D} \rightarrow \infty$ and $\mathscr{D} \rightarrow 0$ :

(a) $\mathscr{D} \rightarrow \infty \quad$ [UV $D>2$; IR $D<2]$ : in this case Eq.(3.17) is reduced to $\Delta \psi=0$, i.e., to the equation of a sourceless, stationarity probability amplitude. Whatever the field described by the potential $\mathcal{U}$, it is no longer feeled by the particle: this is nothing but the property of asymptotic freedom, which is already provided in quantum field theories by non abelian fields. This remark is particularly relevant for $D>2$, since in this case this is the UV (i.e. 
small length-scale, high energy) behavior, and we shall recall hereafter that the principle of scale relativity leads one to introduce a scale-dependent generalized fractal dimension $D(r)>2$ for virtual quantum trajectories considered at scales smaller than the Compton length of the electron.

(b) $\mathscr{D} \rightarrow 0$ [UV $D<2$; IR $D>2$ ] : Eq.(3.17) becomes completely degenerate ( $\vartheta \psi=0$ ). Physics seems to be impossible under such a regime. This result is also in agreement with what is known about the quantum-classical transition. Indeed one finds, when trying to translate the behavior of typical quantum paths in terms of fractal properties [1-7], that their fractal dimension quickly jumps from $D=2$ (quantum) to $D=1$ (classical) when the resolution scale $r$ becomes larger than the de Broglie scale. This fast transition actually prevents the domain $1<D$ $<2$ to be achieved in nature (at the level of fundamental laws).

(iii) $\nabla(\delta \mathscr{D})=0$ or $\nabla(\delta \mathscr{D})<<1$ : in this case, which corresponds to a slowly varying diffusion coefficient in the domain considered, or, at the limit, to a diffusion coefficient depending on time but not on position, the right -hand side of Eq.(3.16) vanishes, so that it may still be integrated, yielding:

$$
\left[\frac{\mathscr{U}}{2 m<\mathscr{D}>}+a+\delta \mathscr{D}(\nabla \ln \psi)^{2}\right] \psi=\mathscr{D} \Delta \psi+i \frac{\partial \psi}{\partial t},
$$

where $a$ is a constant of integration. Assuming that $\delta \mathcal{D} / \mathcal{D}$ remains $<<1$, the effect of the term $\delta \mathscr{D} \psi(\nabla \ln \psi)^{2}$ which is in addition to the standard Schrödinger equation and the effect of $\mathcal{D}$ being a function of $x$ and $t$ can be treated perturbatively. One gets an equation of the form

$$
\left[\frac{\mathcal{U}(x, t)}{2 m}+a+b \delta \mathcal{D}(x, t)\right] \psi=\left\langle\mathcal{D}>^{2} \Delta \psi+i<\mathcal{D}>\frac{\partial \psi}{\partial t},\right.
$$

where $a$ and $b$ are constants. Hence the effect of the new terms amounts to changing the form of the potential in the standard Schrödinger equation. Such a behavior could be of interest in the perspective of a future development of a field theory based on the concept of scale relativity and fractal space-time. Indeed, fluctuations in the fractal space-time geometry are expected to imply fluctuations $\delta \mathcal{D}(x, t)$ of the diffusion coefficient (in a way which remains to be described), which in turn will play the role of a potential in Eq. (3.19).

Let us conclude by a brief comment about the complete equation (3.16). In the particular cases considered above, the statistical interpretation of the wave function $\psi$ in terms of $\rho=\psi \psi^{\dagger}$ giving the probability of presence of the particle remains correct, since the imaginary part of the Schrödinger equation is the equation of continuity (see Ref [14] on that point and on the fact that we do not need to write the Fokker-Planck equations in our derivation of Schrödinger's equation). But this may no longer be the case for the general equation, since we took for our definition of $\psi$ in Eq. (3.12) the simplest possible generalization, which may not be the adequate one. A more complete approach will be presented in a forthcoming work, a task which is revealed necessary in particular for applications of our method to chaotic dynamics (see Sec.6 below).

\section{LORENTZIAN SCALE-RELATIVITY}

As we shall now see, the study of a generalization of quantum mechanics to $D>2$ was not unwarranted, since 
such a behavior is actually a possible consequence of the principle of scale relativity. Assume indeed that we know nothing about the laws of scale transformations (contractions and dilations) and that we want to derive them from the principle of relativity. We have seen that quantum mechanics corresponds to fractal dimension 2 of trajectories, i.e., to a curvilinear length integrated along a quantum path which changes under a scale transformation $r \rightarrow r^{\prime}$ as:

$$
\ln \frac{\mathcal{L}\left(r^{\prime}\right)}{\mathcal{L}_{\mathrm{O}}}=\ln \frac{\mathcal{L}(r)}{\mathcal{L}_{\mathrm{O}}}+\mathbb{V} \delta(r) \quad, \quad \delta\left(r^{\prime}\right)=\delta(r)
$$

where we have set $\mathbb{V}=\ln \left(r / r^{\prime}\right)$. From the purely mathematical point of view, this is the structure of the Galileo group, as confirmed by the law of composition of dilations, $\mathbb{V}^{\prime \prime}=\mathbb{V}+\mathbb{V}^{\prime}$. But the general problem to which (4.1) is a solution amounts to finding the laws of linear transformation of fields in a scale transformation which satisfy the principle of scale relativity. This means finding four quantities, $A(\mathbb{V}), B(\mathbb{V}), C(\mathbb{V})$, and $D(\mathbb{V})$, such that

$$
\ln \frac{\varphi\left(r^{\prime}\right)}{\varphi_{\mathrm{o}}}=A(\mathbb{V}) \ln \frac{\varphi(r)}{\varphi_{\mathrm{o}}}+B(\mathbb{V}) \delta(r) \quad ; \quad \delta\left(r^{\prime}\right)=C(\mathbb{V}) \ln \frac{\varphi(r)}{\varphi_{\mathrm{o}}}+D(\mathbb{V}) \delta(r)
$$

The general solution to this "special relativity problem" is the Lorentz group [15], while the Galileo group, favored up to now in today's physics (concerning scale laws), is only a peculiar solution. We send the reader to Refs [1,14-16] for a more detailed development of these ideas. We shall simply here sum up and compare explicitly the various fundamental rules of present quantum physics (left side in following equations) and their scale-relativistic generalization (right), in the simplified case of pure scale laws (see [1, 15] for general expressions).

Scale dependence of the fractal "curvilinear" length:

$$
\begin{gathered}
\mathcal{L}=\mathcal{L}_{\mathrm{O}}\left(\lambda_{\mathrm{o}} / r\right)^{\delta} \\
\delta(r)=\operatorname{cst} \quad ; \quad \delta(r)=\frac{1}{\sqrt{1-\ln ^{2}\left(\lambda_{\mathrm{o}} / r\right) / \ln ^{2}\left(\lambda_{\mathrm{o}} / \mathbb{A}\right)}} .
\end{gathered}
$$

Scale dependence of coupling constants:

$$
\alpha^{-1}(r)=\alpha^{-1}\left(\lambda_{o}\right)+\beta_{\mathrm{o}} \ln \frac{\lambda_{o}}{r}+\ldots \quad ; \quad \text { unchanged in terms of length-scale }
$$

Law of composition of dilatations (virtual relation):

$$
\ln \frac{r^{\prime}}{\lambda_{\mathrm{o}}}=\ln \frac{r}{\lambda_{\mathrm{o}}}+\ln \rho \quad ; \quad \ln \frac{r^{\prime}}{\lambda_{\mathrm{o}}}=\frac{\ln \left(r / \lambda_{\mathrm{o}}\right)+\ln \rho}{1+\frac{\ln \rho \ln \left(r / \lambda_{\mathrm{o}}\right)}{\ln ^{2}\left(\lambda_{\mathrm{o}} / \AA\right)}}
$$

Compton relation: 


$$
\ln \frac{m}{m_{\mathrm{O}}}=\ln \frac{\lambda_{\mathrm{O}}}{\lambda} \quad, \text { with } \lambda_{\mathrm{o}} m_{\mathrm{o}}=\hbar / c \quad ; \quad \ln \frac{m}{m_{\mathrm{O}}}=\frac{\ln \left(\lambda_{\mathrm{o}} / \lambda\right)}{\sqrt{1-\frac{\ln ^{2}\left(\lambda_{\mathrm{O}} / \lambda\right)}{\ln ^{2}\left(\lambda_{\mathrm{o}} / \mathbb{\Lambda}\right)}}}
$$

Heisenberg's inequality:

$$
\ln \frac{\sigma_{p}}{p_{\mathrm{o}}} \geq \ln \frac{\lambda_{\mathrm{o}}}{\sigma_{x}} \quad, \text { with } \lambda_{\mathrm{o}} p_{\mathrm{o}}=\hbar \quad ; \ln \frac{\sigma_{p}}{p_{\mathrm{o}}} \geq \frac{\ln \left(\lambda_{\mathrm{o}} / \sigma_{x}\right)}{\sqrt{1-\frac{\ln ^{2}\left(\lambda_{\mathrm{o}} / \sigma_{x}\right)}{\ln ^{2}\left(\lambda_{\mathrm{o}} / \Lambda\right)}}}
$$

Scale invariant:

$$
\delta=\text { constant } \quad ; \quad d \sigma^{2}=\mathbb{C}_{\mathrm{o}}^{2} d \delta^{2}-\frac{d \mathcal{L}^{2}}{\mathcal{L}^{2}} \quad, \text { with } \mathbb{C}_{\mathrm{o}}=\ln \left(\frac{\lambda_{\mathrm{O}}}{\mathbb{\Lambda}}\right)
$$

In these equations, valid for $r \lesssim \lambda_{\mathrm{o}}, \lambda_{\mathrm{o}}$ is the Compton length of the electron (or its Lorentz transform), and there appears a fundamental length-scale $\mathbb{\Lambda}$, that is invariant under dilations and contractions (in a way similar to the invariance of the velocity of light under motion transformations), and which can be naturally identified with the Planck length, $\left(\hbar G / c^{3}\right)^{1 / 2}$, (see $[1,14,15]$ for more detail).

\section{APPLICATION IN MICROPHYSICS: MASS-CHARGE SPECTRUM OF PARTICLES}

One of the most encouraging result of the theory of scale relativity in the microphysical domain is its ability to provide us with a possible mechanism of generation of the mass spectrum of elementary particles. This mechanism is able to generate the mass and charge spectrum of elementary fermions in terms of the electron mass and of the electron charge. A first description of this mechanism has been given in [14]. We want to briefly recall here its principle, but also to correct an error that remained in this first presentation and in ref. [16]. Our result is illustrated in Fig. 1.

It is now clear from the above developments that standard quantum mechanics is provided to us only by the twin Wiener-Markov process whose fractal dimension is 2. On the other hand, the requirement of scale covariance (leading to a reinterpretation of the Planck length scale as a limiting, lowest scale in nature, invariant under dilations) implies to introduce a generalized, scale-dependent "fractal dimension" $D(r)=1+\delta(r)$ becoming larger than 2 for scales smaller than the Compton length of the electron (Eq. 4.3b). However, $D$ is not the only quantity that becomes scale dependent in Eq. (3.6) precisely beyond this scale. This is also the case of the "diffusion coefficient" $\mathcal{D}$, since it depends on the electron mass, that becomes itself scale-dependent because of radiative corrections. We have found [14] that the scale dependence of the electron mass (self-energy) due to QED radiative corrections partly cancels the scale dependence of the diffusion coefficient which comes from $D \neq 2$, in such a way that the effective fractal dimension remains close to 2 . Namely, setting $\delta t / \tau \approx r / \lambda_{\mathrm{e}}$ (valid for $r \ll<\lambda_{\mathrm{e}}$, where $\lambda_{\mathrm{e}}$ is the Compton length of the electron), Eq. (3.6) writes for an electron (in the relativistic case) 


$$
<d \xi_{ \pm} i d \xi_{ \pm}>= \pm \frac{\hbar}{m_{\mathrm{e}}} \delta_{i j} d s \quad \mathrm{x} \frac{m_{\mathrm{e}}}{m(r)}\left(r / \lambda_{\mathrm{e}}\right)^{(2 / D(r))-1}
$$

with $D(r)=1+\delta(r)$ as given by Eq.(4.3b), and $m(r)=m_{\mathrm{e}}\left[1+\left(3 \alpha_{\mathrm{e}} / 2 \pi\right) \ln \left(\lambda_{\mathrm{e}} / r\right)\right]$ to lowest order. The remarkable result is that the scale-dependent corrective term to the pure Markov-Wiener process in this equation vanishes precisely at the muon scale $(\approx 100 \mathrm{MeV})$, and that reversely, requiring such a cancellation allows one to predict the muon mass (see [14] for a more precise calculation).

Beyond the muon scale, the variation of the running charge is given by:

$$
\frac{\alpha_{\mathrm{e}}}{\alpha(r)}=1-\frac{2 \alpha_{\mathrm{e}}}{3 \pi}\left[\sum_{i=0}^{n}{Q_{\mathrm{i}}}^{2} \mathbb{V}-\sum_{i=0}^{n}\left(Q_{\mathrm{i}}{ }^{2} \mathbb{V}_{\mathrm{i}}\right)\right] \text {. }
$$

where $\alpha_{\mathrm{e}}(\approx 1 / 137.036)$ is the low energy fine structure constant, $n$ is the number of elementary pairs of fermions of dimensionless charges $Q_{\mathrm{i}}$ and of Compton length $\lambda_{\mathrm{i}}=\hbar / m_{\mathrm{i}} c>r$, and where we have set $\mathbb{V}=\ln \left(\lambda_{\mathrm{e}} / r\right)$ and $\mathbb{V}_{\mathrm{i}}$ $=\ln \left(\lambda_{\mathrm{e}} / \lambda_{\mathrm{i}}\right)$. This formula is to lowest order and neglects threshold effects (see [14] for an improved treatment).

Now, we deduced in Refs $[14,16]$ the running mass from the running charge from the relation $\mathrm{m} / \mathrm{m}_{\mathrm{e}}=$ $\left(\alpha / \alpha_{\mathrm{e}}\right)^{9 / 4}$. But this relation is valid only between the electron and muon scales, so that the scale-relativistic increase in Eq. (5.1) is no longer cancelled by the variation of mass beyond the muon energy, contrarily to what was expected in Ref. [14]. However, the fact that the QED variation of $\left(\alpha / \alpha_{\mathrm{e}}\right)^{k}$, as deduced from Eq. (5.2), with $k$ bestfitted at values $2.25-2.75$, coincides very closely with our scale relativistic correction $\left(\propto \mathbb{V}^{3}\right)$ on the whole electron mass-top mass range, remains true and remarkable. It allows one, reversing the argument, to obtain the full masscharge spectrum of elementary fermions in terms of only the mass and the charge of the electron (that are themselves related in a more evolved version of the theory, see [16]) and the free parameter $k$, as illustrated in Fig. 1. Our interpretation of this result is that the electric charge is a geometric (in the enlarged meaning of nondifferentiable, fractal geometry) property of the electron (itself identified with the fractal geodesics of a nondifferentiable space-time), and that the virtual particle pairs that contribute to its variation are themselves manifestations of these geometric structures. In such a view, the discretization of the mass of elementary particles is a direct consequence of the quantization of charge. A more complete understanding of these relations, including the meaning of the above parameter $k$, can be expected from the future development of a theory of scale relativity generalized to non linear scale transformations and including motion / scale coupling (see [16] for a first attempt in this direction). 


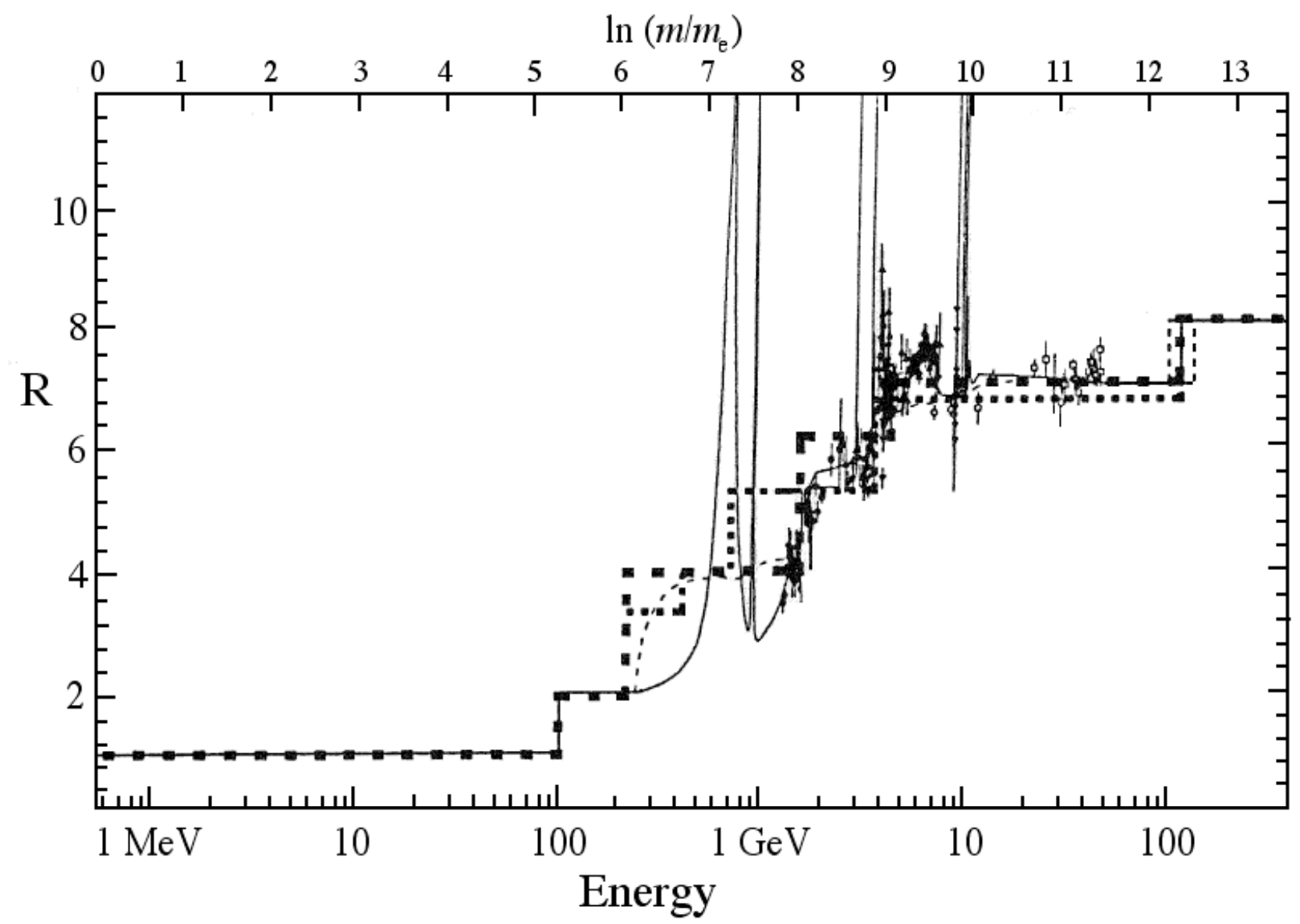

Fig. 1. Comparison of our prediction for the variation with mass scale of the sum of charge squares for elementary fermions (bold broken lines) to the observed $R$ ratio (points with error bars: experimental; thin continuous and broken line: respectively parametrization and first order QCD calculation of $R$, by Burkhardt et al [19]). The $R$ ratio of the $e^{+} e^{-}$annihilation cross sections into hadrons and muon-antimuon pairs amounts to lowest order to the sum of the square of charges of elementary fermions. This figure is adapted from Fig.3 of Ref [19], by adding the leptonic e, $\mu, \tau$ contributions to the hadronic ones, and by adding a top quark contribution of mass $120 \pm 30 \mathrm{GeV}$ [20] (that agrees within error bars with the more recently measured mass $174 \pm 17 \mathrm{GeV}$ ).

This is illustrated in Fig.1, in which we compare our theoretical prediction for the scale variation of the sum of the square of charges of elementary fermions to the experimentally observed variation.

\section{APPLICATION TO CHAOTIC DYNAMICS}

Chaos (often defined as extreme sensibility to variation of initial conditions) is relevant in a huge variety of natural systems. Although various methods of analysis have been coined to describe the development of chaos, all of them have up to now struck against the unpassable barrier of unpredictability at large time scales. However, in many systems where chaos arises, spatial and temporal structures are observed experimentally; such structures are in some few cases found or confirmed in numerical simulations, but very rarely understood or predicted from a fundamental theory.

We have suggested a method for tackling this problem [1]. The basis of this method is the observation that, when looked at time scales very large with respect to the "chaos time" (or inverse Lyapunov exponent $\tau$ ), chaotic trajectories become nondifferentiable, fractal trajectories. Hence the theory described in Sec.3, which would apply 
exactly to quantum phenomena (the nondifferentiability being unbroken at small scale in this case), can be also applied as an approximation to chaotic phenomena (this can be only a large time scale approximation, since differentiability is recovered at small scales in this case).

The first step then consists in giving up the concept of well-defined trajectory at large time scales, and in introducing families of virtual trajectories $[1,4]$. The true trajectory is one random realization among the infinite number of trajectories of the family. Such families are now characterized by a probability density $\rho$. In order to describe them, we assume, once the chaos developed, that the virtual trajectories evolve following a diffusion process, characterized by some diffusion coefficient $\mathcal{D}$. The study of Sec.3 applies to this problem and, provided it allows a Lagrangian approach, we can write a complex Euler-Lagrange equation such as (3.10).

Let us recall how one can apply this method to the formation of the Solar System [1]. Current models assume that the planets formed from a primordial nebula of about $10^{-2}$ solar mass; after the collapse of the nebula into a disk, the particles fall into the equatorial plane and accrete by collisions (see e.g.[21]). At the end of this period of formation, the motion of planetesimals is expected to be both of purely gravitational origin and strongly chaotic. So we approximate its description by considering a point particle in the field of the Sun, subjected to Brownian motion due to the effect of all the other planetesimals. The corresponding equation writes in the stationary case

$$
\mathcal{D} \Delta \psi+\frac{1}{2 m \mathscr{D}}\left[E+\frac{G M m}{r}\right] \psi=0 \text {. }
$$

where $r$ is now the radial distance to the Sun. As shown in Sec.3, this equation remains a good first order approximation even if the diffusion coefficient is no longer a constant, as expected in the case considered here. A diffusion coefficient slowly variable, or $\propto 1 / r$, will yield essentially the same equation, just changing the constants. The result, which has been described in Ref [1], is summarized in Fig. 2.

We predict that the probability densities of the distances of planets to the Sun are confined to quantized, definite regions given by functions ressembling the hydrogen atom orbitals [1], in agreement with their observed positions. The theory also explains the distribution of eccentricities and angular momentum in the planetary system. We have plotted in Fig.2 the expected average distances versus the observed ones. Two laws are found, one for the inner telluric planets, the other for the outer solar system, corresponding (as expected) to two different values of the average diffusion coefficient. However, it is remarkable that these two laws collapse into one, since the inner system as a whole corresponds to the first "orbital" of the outer system (with Earth, the most massive planet in the inner system, at peak), as schematized by the broken lines of Fig. 2. 


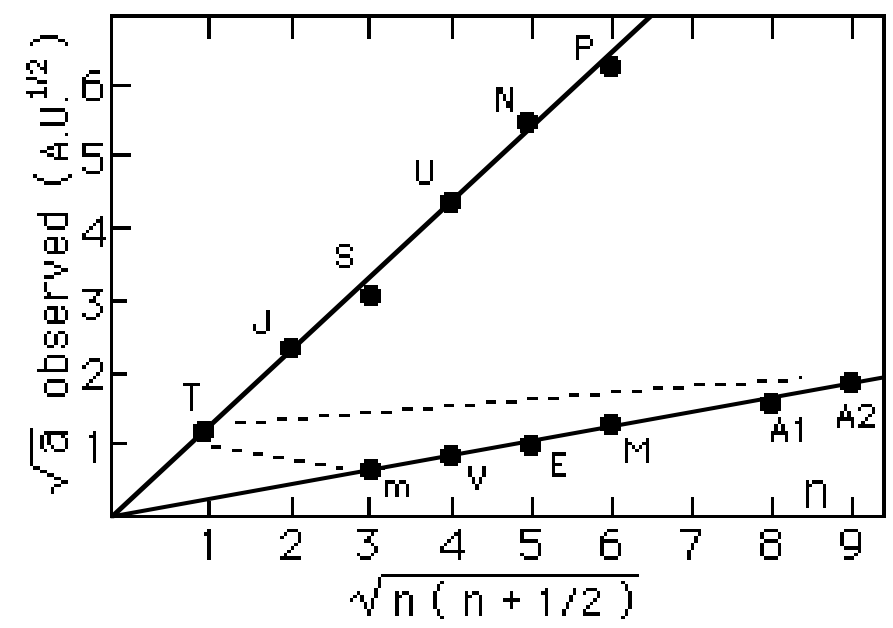

Fig. 2. Comparison of the observed average distances of planets to the sun to our prediction. T stands for the average distance of inner telluric planets. A1 and A2 are for the two main peaks in the distribution of asteroids in the asteroid belt. We predict the possible existence of a new small planet at $n=2(0.185$ A.U.) in the inner system, while $n=1$ is too close to the Sun and $n=7$ is destroyed by resonance with Jupiter.

Note also that the mass distribution in both outer and inner systems is in qualitative agreement with the probability distribution in such orbitals. This suggests a hierarchical process at work and allows one to make predictions about the mass distribution in the very distant outer region of the Solar System.

\section{SUMMARY AND PROSPECT}

We have shown in the present paper that the use of our new complex "covariant derivative" transforms Newton's equation of dynamics into Schrödinger's equation in the quantum mechanical case $(D=2$ and constant diffusion coefficient). Moreover, the scale-relativistic method allows us to suggest a generalization of the quantum theory at very high energies. Indeed, the structure of the present renormalization group is that of the Galileo group, while, as is already the case concerning the laws of motion, the natural structure of the group of scale transformations is Lorentz's. The introduction of a Lorentzian structure for the group of dilations at small lengthscales has interesting consequences concerning the asymptotic behaviour of the quantum theory (reinterpretation of the Planck length and time as limiting, unpassable scales, invariant under dilations and contractions; solution to divergence problems) and allows one to suggest a mechanism for the origin of the mass spectrum of elementary particles.

Concerning chaotic dynamics, we argue that the same method applies to developed chaos for time-scales very large compared with the inverse Lyapunov exponent, i.e., beyond the horizon of predictability. Indeed, in this situation we have suggested to replace deterministic trajectories by families of virtual trajectories which can be approximated by non-differentiable curves, and to give up well-defined positions on behalf of their probability densities. This approach leads to new "quantum-like" complex equations for chaotic systems, whose solutions yield structures in terms of peaks of probability density (but the interpretation is different from that of quantum mechanics).

Let us conclude by a brief prospect on the future development of this theory. Concerning the microphysical 
domain, additional work is needed at three levels [16]: the relation from fractal space-time to the trajectories seen as its geodesics; the passage to relativistic quantum mechanics, including the account of spin; the generalization of scale relativity to non-linear scale transformations and inclusion of fields. Concerning chaotic dynamics, we shall improve and detail our analysis of the Solar System problem and attempt, in studies to come, to generalize our method. Then its application to various problems of structuration possibly linked to chaos, in particular in extragalactic astrophysics and cosmology, will be considered.

Acknowledgements- It is a pleasure to thank Drs. A. LeMéhauté and F. Héliodore for interesting discussions, M.S. El Naschie for communication of results prior to publication, and G. Marshall for his invitation in this colloquium.

\section{REFERENCES}

1. L. Nottale. Fractal Space-Time and Microphysics: Towards a Theory of Scale Relativity. World Scientific (1993).

2. L. Nottale and J. Schneider. Fractals and Non Standard Analysis, J. Math. Phys. 25, 1296-1300 (1984).

3. G.N. Ord. Fractal space-time: a geometric analogue of relativistic quantum mechanics, J. Phys. A: Math. Gen. 16, 1869-1884 (1983).

4. L. Nottale. Fractals and the quantum theory of space-time, Int. J. Mod. Phys. A4, 5047-5117 (1989).

5. R.P. Feynman and A.R. Hibbs. Quantum Mechanics and Path Integrals. MacGraw-Hill (1965).

6. L.F. Abbott and M.B. Wise. Dimension of a quantum mechanical path, Am. J. Phys. 49, 37-39 (1981).

7. E. Campesino-Romeo, J.C. D’Olivo, and M. Socolovsky. Hausdorff dimension for the quantum harmonic oscillator, Phys. Lett 89A, 321-324 (1982).

8. B. Mandelbrot. Les Objets Fractals. Flammarion, Paris (1975); B. Mandelbrot. The Fractal Geometry of Nature. Freeman, San Francisco (1982).

9. D. Sornette. Brownian representation of fractal quantum paths, Euro. J. Phys. 11, 334-337 (1990).

10. M.S. El Naschie. On the uncertainty of information in quantum space-time, Chaos, Solitons and Fractals, 2, 91-94 (1992); Multidimensional Cantor sets in classical and quantum mechanics, Chaos, Solitons and Fractals 2, 211-220 (1992); A note on Heisenberg's uncertainty principle and Cantorian space-time, Chaos, Solitons and Fractals, 2, 437-439 (1992); On certain infinite dimensional Cantor Sets and the Schrödinger Wave, Chaos, Solitons and Fractals, 3, 89-98 (1993).

11. K.W. Höfer. Differential geometry on fractal space-time, Preprint University of Freiburg THEP 91/6 (1991).

12. E. Nelson. Derivation of the Schrödinger Equation from Newtonian Mechanics, Phys. Rev. 150, 1079-1085 (1966).

13. E. Nelson. Quantum Fluctuations. Princeton Univ. Press (1985).

14. L. Nottale. Scale Relativity, Fractal Space-Time and Quantum Mechanics,Chaos, Solitons and Fractals, 4, 361-388 (1994)

15. L. Nottale. The Theory of Scale Relativity, Int. J. Mod. Phys. A7, 4899-4936 (1992).

16. L. Nottale. Scale-Relativity: First Steps toward a Field Theory, in "Relativity in General", E.R.E. 93 (Spanish Relativity Meeting), Ed. J. Diaz Alonso and M. Lorente Paramo, p.121, Editions Frontières (1995).

17. B.B. Mandelbrot \& J.W. Van Ness. Fractional Brownian motions, fractional noises and applications. SIAM Review 10, 422.

18. A.J. Buras, J. Ellis, M.K. Gaillard, D.V. Nanopoulos. Aspects of the grand unification of strong, weak and electromagnetic interactions, Nucl. Phys. B135, 66-92 (1978); D.V. Nanopoulos \& D.A. Ross, Limit on the number of flavours in grand unified theories from higher order corrections to fermion masses, Nucl. Phys. B157, 273-284 (1979).

19. H. Burkhardt, F. Legerlehner, G. Penso, C. Vergegnassi. Uncertainties in the hadronic contribution to the QED vacuum polarization, Z. Phys. C-Particles and Fields 43, 497-501 (1989).

20. P. Langacker, M. Luo, \& A.K. Mann. High precision electroweak experiments: A global search for new physics beyond the Standard Model, Rev. Mod. Phys. 64, 87-192 (1992).

21. T. Encrenaz, J.P. Bibring and M. Blanc. The Solar System, Springer-Verlag, Berlin (1991). 